\title{
Farmacoeconomia dei COXIB nella patologia osteoarticolare: revisione della letteratura
}

Pierluigi Russo*, Luca Degli Esposti§, Alessandro Capone§, Ezio Degli Esposti ${ }^{\circ}$, Luciano Caprino*

\section{ABSTRACT}

A new class of anti-inflammatory agents, the selective inhibitors of cyclooxygenase-2 (COXIBs), has been recently introduced into the market for the treatment of osteoarthritis and reumatoid arthritis. Randomized and controlled clinical trials showed a similar efficacy and a better tolerability profile of COXIBs compared with conventional non-steroidal anti-inflammatory drugs (NSAIDs). The aim of this study was to perform a scientific literature review relating to the economic impact produced by COXIBs' introduction. The research of references included the following databases: MEDLINE, EMBASE and the NHS (Economic Evaluation Database) of the York University. A total of 67 in extenso pubblications have been extracted. Of these 13 papers having the specific objective to evaluate the economic implications of COXIBs in comparison to conventional NSAIDs was analysed. In ten cases (77\%), cost-effectiveness analyses were performed. The European context was considered in eight cases (62\%), while that of North America and Asia were investigated in four and one case, respectively. The analysis of costs took always into account direct costs of the management of arthritis exclusively (drugs, and resources associated with the treatment of gastrointestinal side effects). Indirect and intangible costs were never considered.

The results of this review highlight that the higher tollerability profile of COXIBs may generate a cost-saving. This cost-saving seems to be basically due to the reduced frequency of gastroprotective agents coprescription and also to a lesser appearance of severe gastrointestinal side effects compared with conventional NSAIDs. Besides the disease management improving, the cost-saving associated with COXIBs can completely or partially offset the net increase of expense induced by their higher price of purchase.

Farmeconomia e percorsi terapeutici 2003; 4 (Suppl 1): 5-13

\section{INTRODUZIONE}

Le malattie osteoarticolari comprendono un insieme di condizioni patologiche progressivamente invalidanti, che in Italia si manifestano con una prevalenza compresa tra il $10 \%$ e il $18,3 \%[1,2]$. In altre nazioni industrializzate sono stati rilevati simili dati di prevalenza, con valori intorno al $15 \%$ [3].

Tra le malattie osteoarticolari, l'artrosi e l'artrite reumatoide sono quelle più rappresentative. L'artrosi è la più comune reumoartropatia e la più frequente causa di invalidità negli anziani, coinvolgendo l'articolazione dell'anca (coxartrosi) nel 7,7\% della popolazione con età maggiore di 64 anni, le articolazioni della mano nel $14,9 \%$ e l'articolazione del ginocchio (gonartrosi) nel 29,9\% [4]. Riferendo tali percentuali di prevalenza alla popolazione italiana, nella medesima fascia di età, si deduce che circa 5 milioni di persone siano affette da artrosi sintomatica.
L'artrite reumatoide è una malattia sistemica cronica, caratterizzata da ripetuti fenomeni infiammatori che colpiscono le cartilagini articolari e che svolgono un ruolo centrale nella evoluzione del danno osteocartilagineo. Sebbene l'artrite reumatoide abbia una prevalenza che è circa 10 volte inferiore quella dell' artrosi (ossia circa 1'1\% negli USA), presenta dei costi per paziente che sono superiori a quelli dell'artrosi [5]. Ciò è da mettere in relazione con l'esordio della malattia, che nell' $80 \%$ dei casi coinvolge la fascia di età compresa tra i 35 e i 50 anni, con il coinvolgimento contemporaneo di più articolazioni (poliartrite cronica) e con una evoluzione progressivamente invalidante.

Il trattamento di queste patologie osteoarticolari ha generalmente un duplice obiettivo: sintomatico, volto ad alleviare il dolore, e di rallentamento della progressione del danno osteocartilagineo, attraverso la riduzione di eventuali fenomeni infiammatori. Tali
* Dipartimento di Fisiologia Umana e Farmacologia, Università "La Sapienza", Roma $\S$ Clicon S.r.l., Health,

Economics, and

Outcome Research,

Ravenna

- Dipartimento di valutazione dell'efficacia clinica, Azienda Unità Sanitaria Locale 110 di Ravenna 
obiettivi terapeutici vengono perseguiti prevalentemente mediante la somministrazione di farmaci anti-infiammatori non steroidei (FANS) convenzionali.

Tuttavia, l'impiego di tale classe di farmaci è associato ad un rilevante rischio di reazioni avverse a carico dell' apparato gastrointestinale, anche in rapporto con la concomitante presenza di altri fattori di rischio (età maggiore di 65 anni, sesso maschile, abitudine al fumo, presenza di precedenti ospedalizzazioni per eventi gastrointestinali, prescrizione di cortisonici, numero di differenti FANS impiegati) [6,7]. Negli Stati Uniti si stima che le ospedalizzazioni per complicanze gastrointestinali coinvolgano oltre 100.000 pazienti ogni anno e si associno a circa 16.500 decessi, con un costo per evento gastrointestinale da FANS variabile tra 1.200 e 6.127 US\$ [5].

In Italia, utilizzando un questionario somministrato a 51 medici di medicina generale, è stata effettuata una analisi del costo medio di ogni evento gastrointestinale verificatosi in corso di trattamento con FANS, in un periodo di osservazione di 180 giorni [8]. Il costo è risultato variabile da 491,48 euro (per ulcerazioni che non hanno richiesto l'ospedalizzazione) a 10.809,34 euro (per eventi di perforazione/ ostruzione gastrointestinale o sanguinamento massivo). In termini di costo iatrogeno, per ogni unità monetaria spesa per la terapia con FANS è necessario considerare un aggravio di costo, destinato a sostenere la spesa per la prevenzione ed il trattamento degli eventi avversi FANS-indotti, che è compreso tra $2,3 \mathrm{e}$ $2,8[1]$.

Allo stato attuale, con l'intento di prevenire la comparsa di eventi avversi gastrointestinali, al trattamento con FANS è associata frequentemente la contemporanea prescrizione di farmaci gastroprotettori (inibitori della pompa protonica, H2-antagonisti, misoprostolo, sucralfato e antiacidi). Dai dati di letteratura emerge che la frequenza della co-prescrizione per farmaci gastroprotettori varia tra il 20-26\% nei Paesi anglosassoni $[9,10]$ e il 42-50\% in Francia, Spagna e Italia [1, 11-12].

Recentemente una nuova classe di farmaci analgesici-anti-infiammatori, gli inibitori specifici della ciclossigenasi di tipo 2 (COXIB: rofecoxib e celecoxib), hanno dimostrato un'efficacia equivalente a quella dei FANS convenzionali di più frequente impiego e un profilo di sicurezza e tollerabilità gastrointestinale superiore [13-17].

Sulla base delle evidenze ottenute nei trial clinici randomizzati, l'introduzione dei COXIB dovrebbe aver avuto un impatto clinico ed economico rilevante sul trattamento delle malattie osteoarticolari. Scopo di questo articolo è quello di effettuare una revisione dei dati di lettera- tura, relativi alle valutazioni farmacoeconomiche che sono state condotte fino ad oggi sui COXIB.

\section{METODOLOGIA}

È stata condotta una ricerca bibliografica sulle pubblicazioni, in lingua inglese e non, censite all'interno delle banche dati MEDLINE (tra $1^{\circ}$ gennaio 1990 e 31 aprile 2003), EMBASE (tra $1^{\circ}$ gennaio 1990 e 31 settembre 2002) e la banca dati del NHS Centre for Reviews and Dissemination dell'Università di York (NHS Economic Evaluation Database; NHS EED).

Per quanto riguarda le prime due banche dati, sono state imputate le parole chiavi: "COXIB" o "rofecoxib" o "celecoxib" o "parecoxib" o "valdecoxib" (con 3.767 record estratti); e le seguenti parole chiavi nel titolo: "cost" o "costs" o "economics" o "pharmacoeconomics" (con 252.575 record estratti). L'incrocio tra queste due selezioni ha portato alla estrazione di 67 record. Nella banca dati NHS EED sono state imputate le seguenti parole chiavi nel titolo: "COXIB" o "rofecoxib" o "celecoxib" o "parecoxib" o "valdecoxib" (con 6 record estratti).

Ogni record ottenuto dalla ricerca bibliografica è stato valutato verificando la rispondenza dei suoi contenuti con l'obiettivo stabilito nel presente articolo. Inoltre, sono state identificate ed eliminate le doppie citazioni. Nell' ambito delle valutazioni economiche, sono stati inclusi solo gli articoli pubblicati in extenso.

\section{RISULTATI}

La ricerca bibliografica ha portato all'identificazione di complessive 67 pubblicazioni (i 6 articoli estratti dalla NHS EED erano compresi anche nella lista derivata dall'interrogazione delle banche dati MEDLINE ed EMBASE). L'analisi degli abstract ha portato all'ulteriore selezione di 13 articoli che avevano il preciso obiettivo di effettuare una valutazione comparativa dei costi sostenuti per il trattamento della patologia osteoarticolare con COXIB rispetto a FANS convenzionali.

Nella Tabella 1 sono riassunte le principali caratteristiche dei 13 studi selezionati [18-30].

In 10 casi $(77 \%)$, si trattava di analisi costoefficacia o di valutazioni che, pur non calcolando il costo per evento evitato o trattato, mettevano in rapporto i costi dei diversi trattamenti farmacologici con le rispettive conseguenze.

Le ricerche hanno considerato prevalentemente il contesto europeo (in 8 casi, pari al $62 \%), 2$ sono state condotte in nazioni del nord 


\section{P. Russo, L. Degli Esposti, A. Capone, E. Degli Esposti, L. Caprino}

Europa (Svezia e Norvegia), 2 in nazioni dell'Europa centrale (Regno Unito e Svizzera) e 4 sono state condotte in nazioni del sud Europa (Spagna e Italia). Delle restanti 5 analisi (pari al $38 \%$ ), 4 sono state condotte in America (USA e Canada) e 1 in Asia (Hong Kong).

La patologia osteoarticolare più frequentemente considerata è stata l' artrosi (7 casi), in 2 casi si è trattato dell' artrite reumatoide, mentre in 4 studi le due patologie sono state analizzate congiuntamente.
L'approccio metodologico seguito per la determinazione dei costi e delle conseguenze del trattamento è stato, nella quasi totalità dei casi (11 studi), quello di tipo modellistico, mediante l'analisi decisionale, mentre solo in 2 casi gli studi presentavano un disegno osservazionale, realizzato a partire da informazioni contenute in database amministrativi. In 10 casi i dati introdotti nel modello, relativi alla comparsa di eventi gastrolesivi indotti da FANS, originavano da trial clinici randomizzati.

\section{Tabella 1}

Caratteristiche generali degli studi inclusi, relativi a valutazioni economiche dei COXIB

\begin{tabular}{|c|c|c|c|c|c|c|c|c|}
\hline Citazione & $\begin{array}{l}\text { Tipo di } \\
\text { analisi }\end{array}$ & $\begin{array}{l}\text { Fonte dei } \\
\text { risultati }\end{array}$ & $\begin{array}{l}\text { Origine dati } \\
\text { introdotti nel } \\
\text { modello }\end{array}$ & Paese & Indicazione & Costi & Conseguenze & $\begin{array}{c}\text { Durata } \\
\text { osservazione }\end{array}$ \\
\hline $\begin{array}{l}{[18]} \\
\text { Haglund and Svarvar, } \\
2000\end{array}$ & $\mathrm{ACE}$ & $\begin{array}{l}\text { Analisi } \\
\text { decisionale }\end{array}$ & $\mathrm{RCT}$ & Svezia & $\begin{array}{l}\text { Artrosi e } \\
\text { AR }\end{array}$ & Sanitari diretti & $\begin{array}{l}\text { Eventi GI } \\
\text { maggiori e } \\
\text { minori }\end{array}$ & 12 mesi \\
\hline $\begin{array}{l}{[19]} \\
\text { Svarvar and Aly, } 2000\end{array}$ & $\mathrm{ACE}$ & $\begin{array}{l}\text { Analisi } \\
\text { decisionale }\end{array}$ & $\begin{array}{l}\mathrm{RCT}+ \\
\text { questionari }\end{array}$ & Norvegia & $\begin{array}{l}\text { Artrosi e } \\
\text { AR }\end{array}$ & Sanitari diretti & $\begin{array}{l}\text { Eventi GI } \\
\text { maggiori e } \\
\text { minori }\end{array}$ & 12 mesi \\
\hline $\begin{array}{l}{[20]} \\
\text { Pellissier et al., } 2001\end{array}$ & $\mathrm{ACE}$ & $\begin{array}{l}\text { Analisi } \\
\text { decisionale }\end{array}$ & RCT & USA & Artrosi & Sanitari diretti & $\begin{array}{l}\text { Eventi GI } \\
\text { maggiori e } \\
\text { minori }\end{array}$ & 12 mesi \\
\hline $\begin{array}{l}{[21]} \\
\text { Soto, } 2001\end{array}$ & $\mathrm{ACM}$ & $\begin{array}{l}\text { Analisi } \\
\text { decisionale }\end{array}$ & $\mathrm{RCT}$ & Spagna & Artrosi & Farmacologici & - & 3 mesi \\
\hline $\begin{array}{l}{[22]} \\
\text { Moore et al., } 2001\end{array}$ & ACE & $\begin{array}{l}\text { Analisi } \\
\text { decisionale }\end{array}$ & $\mathrm{RCT}$ & Regno Unito & Artrosi & Sanitari diretti & $\begin{array}{l}\text { Eventi GI } \\
\text { maggiori e } \\
\text { minori }\end{array}$ & 12 mesi \\
\hline $\begin{array}{l}{[23]} \\
\text { Marshall et al., } 2001\end{array}$ & ACE & $\begin{array}{l}\text { Analisi } \\
\text { decisionale }\end{array}$ & $\mathrm{RCT}$ & Canada & Artrosi & Sanitari diretti & $\begin{array}{l}\text { Eventi GI } \\
\text { maggiori e } \\
\text { minori }\end{array}$ & 12 mesi \\
\hline $\begin{array}{l}{[24]} \\
\text { Zabinski et al., } 2001\end{array}$ & $\mathrm{ACE}$ & $\begin{array}{l}\text { Analisi } \\
\text { decisionale }\end{array}$ & $\begin{array}{l}\mathrm{RCT}+ \\
\text { questionari }\end{array}$ & Canada & $\mathrm{AR}$ & Sanitari diretti & $\begin{array}{l}\text { Eventi GI } \\
\text { maggiori e } \\
\text { minori }\end{array}$ & 6 mesi \\
\hline $\begin{array}{l}{[25]} \\
\text { Chancellor et al., } 2001\end{array}$ & $\mathrm{ACE}$ & $\begin{array}{l}\text { Analisi } \\
\text { decisionale }\end{array}$ & $\begin{array}{l}\mathrm{RCT}+ \\
\text { questionari }\end{array}$ & Svizzera & $\mathrm{AR}$ & Sanitari diretti & $\begin{array}{l}\text { Eventi GI } \\
\text { maggiori e } \\
\text { minori }\end{array}$ & 6 mesi \\
\hline $\begin{array}{l}{[26]} \\
\text { Russo et al., } 2001\end{array}$ & $\mathrm{ACE}$ & $\begin{array}{l}\text { Analisi } \\
\text { decisionale }\end{array}$ & Pratica clinica & Italia & Artrosi & Farmacologici & $\begin{array}{l}\text { Frequenza di } \\
\text { co-prescrizione } \\
\text { di FGP }\end{array}$ & 18 mesi \\
\hline $\begin{array}{l}{[27]} \\
\text { You et al., } 2002\end{array}$ & $\mathrm{ACE}$ & $\begin{array}{l}\text { Analisi } \\
\text { decisionale }\end{array}$ & $\begin{array}{l}\text { RCT + } \\
\text { questionari }\end{array}$ & Hong Kong & $\begin{array}{l}\text { Artrosio } \\
\text { AR }\end{array}$ & Sanitari diretti & $\begin{array}{l}\text { Eventi GI } \\
\text { maggiori e } \\
\text { minori }\end{array}$ & 6 mesi \\
\hline $\begin{array}{l}{[28]} \\
\text { Vargas et al., } 2002\end{array}$ & $\mathrm{ACM}$ & $\begin{array}{l}\text { Analisi } \\
\text { decisionale }\end{array}$ & RCT & Spagna & Artrosi & Farmacologici & - & 6 mesi \\
\hline $\begin{array}{l}{[29]} \\
\text { Weideman et al., } 2002\end{array}$ & $\mathrm{ACM}$ & $\begin{array}{l}\text { Database } \\
\text { amministrativi }\end{array}$ & Pratica clinica & USA & $\begin{array}{l}\text { Artrosi e } \\
\mathrm{AR}^{*}\end{array}$ & Farmacologici & - & 12 mesi \\
\hline $\begin{array}{l}{[30]} \\
\text { Russo et al., } 2003\end{array}$ & - & $\begin{array}{l}\text { Database } \\
\text { amministrativi }\end{array}$ & Pratica clinica & Italia & Artrosi & $\begin{array}{l}\text { Farmacologici } \\
\text { e dei ricoveri } \\
\text { per eventi GI } \\
\text { maggiori }\end{array}$ & $\begin{array}{l}\text { Frequenza di } \\
\text { co-prescrizione } \\
\text { di FGP ed } \\
\text { eventi GI } \\
\text { maggiori }\end{array}$ & 12 mesi \\
\hline
\end{tabular}

Abbreviazioni: $A C E=$ Analisi costo-efficacia; ACM = Analisi costo-minimizzazione; $R C T=$ trial clinico randomizzato; GI = gastrointestinali; $A R=$ artrite reumatoide $; F P=$ farmaci gastroprotettori.

* oltre alle altre condizioni patologiche assistite nell'ambito del Veterans Affairs Healthcare System. 
Nei restanti 3 casi, i dati sono stati rilevati nel contesto della pratica clinica quotidiana: in uno studio, le probabilità di comparsa degli eventi sono state introdotte in un modello decisionale [26]; mentre nei rimanenti 2, è stata effettuata un'analisi diretta degli esiti economici osservati in seguito all'introduzione dei COXIB [29,30].

Nelle valutazioni dei costi sono stati sempre considerati quelli strettamente connessi alla gestione della malattia (costi diretti associati all' acquisto dei farmaci e alle risorse impiegate per la prevenzione/trattamento degli effetti collaterali gastrointestinali). I costi indiretti e quelli intangibili invece sono stati esclusi. In 4 studi, l'imputazione dei costi nei modelli è derivata da dati raccolti con questionari che analizzavano il consumo delle risorse in contesti di medicina specialistica. Nei restanti 7 casi, i costi sono stati calcolati attraverso una simulazione modellistica basata su dati di letteratura. Solo in 2 studi i costi erano quelli effettivamente generati dall'introduzione dei COXIB nella pratica clinica.

Escludendo i 3 studi di costo-minimizzazione, in cui non veniva ovviamente preso in considerazione alcun esito clinico, la tipologia delle conseguenze analizzate è stata nel 90\% dei casi (9 studi) la probabilità di comparsa di eventi gastrointestinali minori (dispepsia) e/o maggiori (ospedalizzazioni per perforazioni, ulcere, sanguinamento e per altri eventi gastrointestinali). In 2 studi, è stata anche analizzata, come esito surrogato della tollerabilità gastrointestinale, la frequenza di co-prescrizione per farmaci gastroprotettori.

L'intervallo temporale analizzato dai diversi studi variava da 3 a 18 mesi, con un $62 \%$ (8 casi) che avevano una durata maggiore o uguale a 12 mesi.

Nelle Tabelle 2 e 3 sono riassunti i risultati delle valutazioni economiche riguardanti i COXIB. Le opzioni confrontate erano quelle relative ai FANS assunti da soli o in combinazione con differenti classi di farmaci gastroprotettori (inibitori della pompa protonica o antagonisti dei recettori $\mathrm{H}_{2} \mathrm{O}$ misoprostolo). Sul parecoxib e sul valdecoxib non è ancora stata effettuata alcuna valutazione economica, per contro ne sono disponibili 7 relative al celecoxib (54\%) e 9 al rofecoxib (69\%).

In nessuno dei modelli riguardanti il celecoxib è stato considerato che una quota dei pazienti potesse ricevere una possibile coprescrizione per farmaci gastroprotettori. Mentre in 3 ricerche relative al rofecoxib, nell'ambito della coorte di pazienti trattati con il COXIB, è stata inclusa anche quella percentuale di coprescrizione per gastroprotettori, rilevata nella pratica clinica (con valori compresi tra il 10\% e il $19,9 \%$ ), e a cui è conseguito un incremento del costo di terapia.

Assumendo come riferimento il costo giornaliero sostenuto per il paziente trattato con soli FANS, l'associazione di un inibitore della pompa protonica ad un FANS costituisce l'opzione che si associa al maggior incremento percentuale del costo atteso, variabile tra un minimo del $78 \%$ (in Hong Kong) e un massimo del $178 \%$ (in Canada).

Segue, con minori incrementi percentuali del costo, la combinazione con gli antagonisti dei recettori $\mathrm{H}_{2}$ (tra il $57 \%$ in Canada e il 136\% in Svizzera). La co-prescrizione di FANS e misoprostolo ha condotto ad un incremento del costo atteso variabile tra il 19\% (in Svezia) e il 103\% (in Svizzera). L'opzione data dall' associazione diclofenac/misoprostolo è stata considerata in 5 valutazioni economiche ed è risultata associata ad incrementi percentuali del costo per paziente, rispetto al trattamento con soli FANS, variabili tra il 2,5-3\% (in Svezia e Svizzera) e il 39\% (in Canada).

Analizzando i risultati ottenuti con i COXIB separatamente per le diverse patologie, nelle valutazioni modellistiche relative all'artrosi (vedi Tabella 2), il celecoxib poteva determinare una riduzione percentuale del costo giornaliero per paziente, rispetto al trattamento con soli FANS, variabile da un minimo del -4\% (in Hong Kong) ad un massimo del -22\% (in Norvegia). In tutti questi casi, il celecoxib risultava associato ad una riduzione del numero di eventi gastrointestinali maggiori e minori, affermandosi sempre come opzione dominante. Inoltre, in due analisi modellistiche $[18,19]$ che hanno confrontato i COXIBs tra loro rispetto all'opzione terapeutica rappresentata dai FANS convenzionali, sono stati ottenuti risultati decisamente contradditori.

Per ciò che concerne il rofecoxib, negli studi in cui non era prevista una percentuale di co-prescrizione per gastroprotettori, a fronte di una maggiore efficacia, il rofecoxib si associava ad un incremento percentuale del costo giornaliero per paziente compreso tra il $4,8 \%$ (in USA) e il $54 \%$ (in UK). Nelle valutazioni economiche realizzate adottando modelli decisionali che consideravano l'impiego di gastroprotettori anche tra i pazienti esposti a trattamento con COXIB, il rofecoxib produceva un incremento del costo giornaliero del $4,4 \%$ (in Canada) [23]. Per quanto attiene alle valutazioni economiche condotte su dati raccolti nella pratica clinica, uno studio ha verificato gli effetti economici generati dal trasferimento di pazienti trattati con FANS più farmaci gastroprotettori verso il trattamento con rofecoxib o rofecoxib più gastroprotettori [26]. Tale scenario terapeutico è stato strutturato sulla base della riduzione della frequenza di 
co-prescrizione per gastroprotettori, registrata nella realtà clinica italiana nei pazienti trattati con rofecoxib.

Nonostante il costo di acquisizione più elevato del COXIB e la presenza della co-prescrizione per gastroprotettori, l'introduzione di rofecoxib si associava ad una riduzione del $6,6 \%$ del costo farmacologico giornaliero sostenuto per paziente. In un successivo studio, gli stessi autori [30] hanno completamente escluso l'approccio modellistico, a favore di una rilevazione diretta degli esiti economici prodotti dall'introduzione del rofecoxib nella pratica clinica corrente.

Poiché i dati erano reali, è stata eseguita una valutazione economica completa, che ha permesso di distinguere tra casi incidenti e prevalenti. Nei casi incidenti, è stato evidenziato che il costo giornaliero del trattamento con rofecoxib è stato del $7.4 \%$ inferiore rispetto a quello sostenuto per il paziente esposto a FANS convenzionali (rispettivamente 1,88 euro e 2,03 euro). Nei casi prevalenti, invece, nonostante la riduzione dell'uso dei gastroprotettori, il costo giornaliero della terapia con rofecoxib è risultata maggiore del $28.1 \%$ (1,87 euro vs. 1,46 euro). Inoltre, sebbene la frequenza di ospedalizzazione per eventi gastrointestinali maggiori fosse sostanzialmente analoga, la spesa giornaliera sostenuta per il paziente esposto a rofecoxib è stata nettamente più bassa, riportando una riduzione percentuale pari all' $88 \%$ di quella registrata nei pazienti trattati con FANS.

Un confronto diretto tra rofecoxib e celecoxib nell' artrosi (oltre che per altre condizioni patologiche), condotto mediante un' analisi di costo-minimizzazione a partire da informazioni contenute in database amministrativi negli USA [29], ha evidenziato un costo giornaliero della terapia istituita con celecoxib del $25,8 \%$ maggiore rispetto al rofecoxib. Gli autori sostengono che questo risultato sia dovuto al maggior numero di somministrazioni quotidiane che, nella pratica clinica, il celecoxib richiede in confronto al rofecoxib [29].

Nel caso dell'artrite reumatoide (vedi Tabella 3), contrariamente a quanto predisposto per l'artrosi, in nessun caso è stata considerata l'opzione rofecoxib. La disponibilità di celecoxib ha determinato una variazione del costo giornaliero per paziente, rispetto al trattamento con soli FANS, variabile da un minimo del -14,5\% (in Svizzera) a un massimo del $+4 \%$ (in Canada). In tutti i casi, il celecoxib è stato associato ad una riduzione del numero di eventi gastrointestinali maggiori e minori. Di conseguenza, allo stato attuale, nell' artrite reumatoide, il celecoxib è risultato l'opzione dominante o comunque quella più efficace, a fronte di un marginale incremento del costo sostenuto per paziente.

\section{DISCUSSIONE}

La revisione della letteratura relativa alle valutazioni economiche dei COXIB, nel trattamento delle patologie osteoarticolari, ha evidenziato la disponibilità di un cospicuo numero di informazioni, che sono state prodotte, nella maggior parte dei casi nei paesi a più alta industrializzazione, in seguito alla commercializzazione dei primi due COXIB (rofecoxib e celecoxib). Tuttavia, proprio perché l'introduzione di questi nuovi anti-infiammatori è molto recente, le valutazioni economiche sono state condotte prevalentemente mediante proiezioni modellistiche. In effetti, soltanto da poco iniziano a diffondersi informazioni sulle reali conseguenze prodotte dall'introduzione dei COXIB nella pratica clinica. $\mathrm{Nel}$ $77 \%$ dei casi (10 pubblicazioni su 13), gli effetti clinici ed economici dei COXIB sono stati stimati facendo ricorso all' analisi decisionale basata su dati di efficacia, rilevati nel contesto di trial clinici randomizzati.

L'interpretazione dei risultati ottenuti mediante la realizzazione di modelli che si limitano a simulare la realtà impone, comunque, una notevole cautela; soprattutto quando i modelli sono criticamente condizionati da dati di probabilità rilevati nell'ambito dei trial clinici randomizzati. Ciò determina un' eventuale sovra/sottostima dei risultati, per effetto della potenziale esclusione di fattori in realtà non trascurabili o per effetto di una loro rilevante variazione quantitativa. Il contesto sperimentale del trial clinico randomizzato obbliga, infatti, il ricercatore in una serie di processi metodologici e decisionali vincolanti che, di fatto, divergono profondamente da quelli che lui stesso avrebbe seguito in condizioni di reale pratica clinica.

Indipendentemente dall'approccio seguito, una valutazione economica dei COXIB dovrebbe prendere in considerazione tre elementi fondamentali: (1) il maggior costo di acquisto dei COXIB rispetto a quello dei FANS convenzionali; (2) le conseguenze economiche derivanti dalla minore incidenza di eventi avversi gastrointestinali (sia minori, che maggiori) nei pazienti trattati con COXIB; (3) la valenza economica della potenziale riduzione, nei pazienti trattati con COXIB, del consumo di farmaci gastroprotettori, impiegati per la prevenzione o per il trattamento della gastrolesività da FANS [31].

Mentre la maggior parte delle valutazioni economiche fin qui descritte (escludendo ov- 

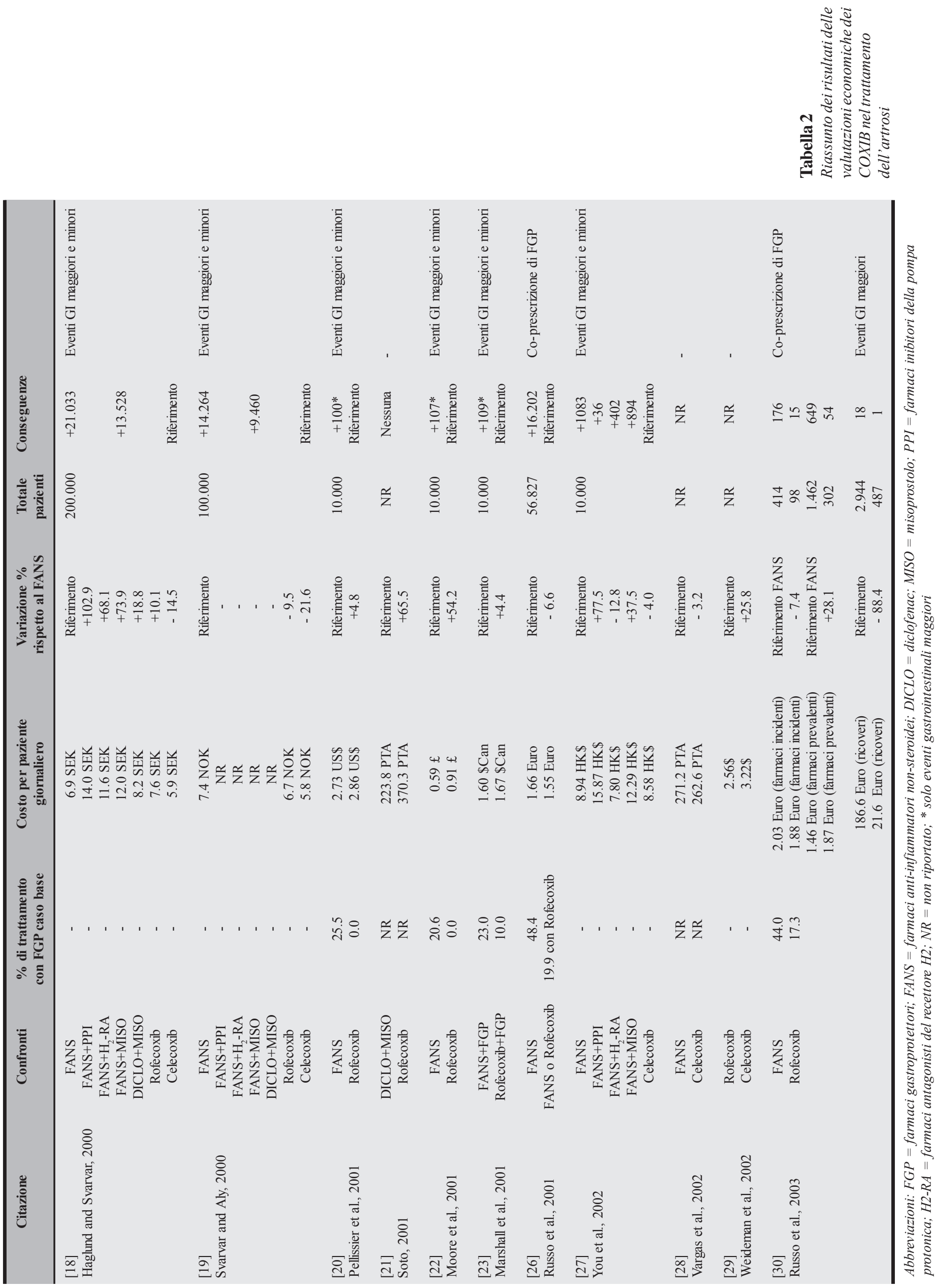


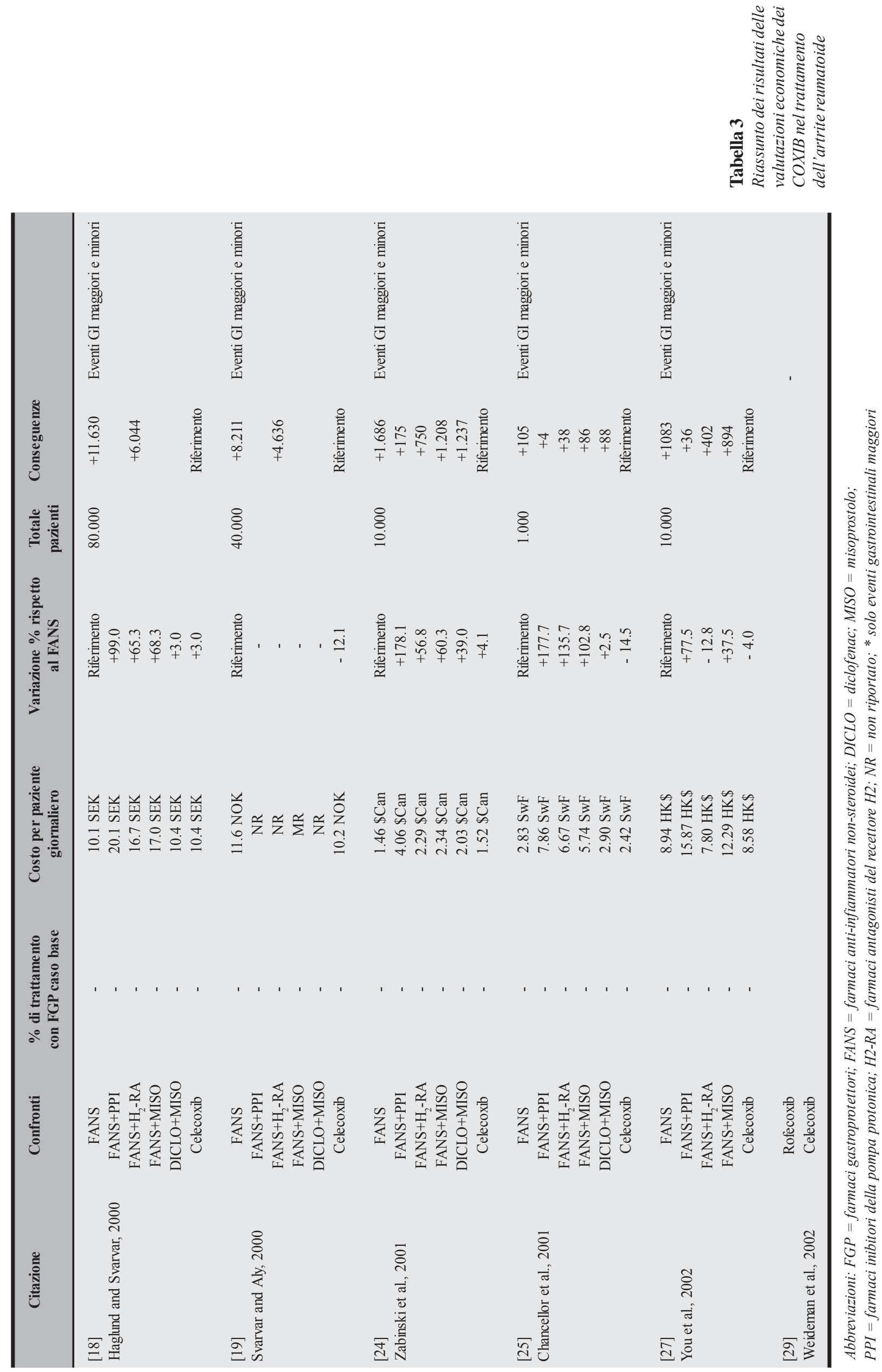


viamente le analisi di costo-minimizzazione) presentavano un approccio orientato al raggiungimento dei primi due obiettivi (i.e. costi farmacologici e risorse usate per il trattamento di eventi avversi gastrointestinali), più disomogeneo è stato l'approccio relativo all'inclusione dei costi generati dalla co-prescrizione per farmaci gastroprotettori. Infatti, dall'analisi comparata degli studi, quest'ultimo elemento di costo è stato considerato solo in quelli che hanno valutato gli effetti economici prodotti dal rofecoxib nel trattamento dell'artrosi [23, 26, 30].

Nella pratica clinica, la scelta di usare farmaci gastroprotettori, anche in pazienti trattati con COXIB, è una considerazione assolutamente imprescindibile, poiché il medico può trovarsi di fronte a pazienti con un profilo di rischio multiplo. In generale, escludere dai modelli di valutazione economica sui COXIB la presenza di una percentuale di co-prescrizione per farmaci gastroprotettori equivale a sovrastimare i reali benefici economici ottenibili con questi farmaci.

Da una precedente revisione della letteratura, realizzata nel contesto di una Consensus Conference sui COXIB [31] è scaturita la definizione del seguente, condivisibile, statement: "studi di valutazione economica condotti con modelli indicano la convenienza dei COXIB se questi non sono associati a farmaci per la profilassi del danno gastrointestinale: rimane da confermare questa osservazione nella pratica clinica". Gli studi pubblicati successivamente iniziano ad evidenziare in modo convincente come - nella pratica clinica - il medico impieghi i modo efficiente i COXIB: tale da massimizzarne i benefici terapeutici, minimizzandone l'impatto economico [30].

In conclusione, i risultati di questa revisione evidenziano come la migliore tollerabilità dei COXIB, rispetto ai FANS convenzionali, possa concretizzarsi in una riduzione dei costi conseguenti sia alla minore frequenza di eventi avversi gastrointestinali, sia alla modificazione del disease management nei pazienti affetti da patologie osteoarticolari (i.e., riduzione della frequenza di co-prescrizione per farmaci gastroprotettori). Tale riduzione dei costi, inoltre, può essere capace di assorbire - in tutto o in parte - l'incremento di spesa derivante dal maggior costo di acquisizione dei COXIB. Ciò contribuisce a consolidare, anche nella prospettiva economica oltre che a quella strettamente farmacologica, l'idea di considerare questi farmaci come una vera e propria alternativa al trattamento con FANS convenzionali delle patologie articolari croniche [32].

\section{BIBLIOGRAFIA}

1. Leardini G. Dimensioni e costi degli effetti indesiderati FANS indotti nel paziente affetto da artrosi in Italia. Reumatismo 2000; 52(4): 223-233.

2. ISTAT 2000. Le condizioni di salute degli italiani. Anno 1999. ISTAT Roma, 2000

3. Badley EM, Crotty M. An international comparison of the estimate effect of the aging of the population of the major cause of disablement, muscoloskeletal disorders. J Rheumatol 1995; 22: 1934-40.

4. Mannoni A, Briganti MP, Di Bari M et al. The epidemiological profile of symptomatic osteoarthritis in older adults: a population based study in Dicomano, Italy. Ann Rheum Dis 2003; in press.

5. Peloso PM, Scheiman JM. The economic implications of cyclooxygenase-2-specific inhibitors. Am J Med 2001; 110(3A):50S-54S.

6. Henry D, Lim LL, Garcia Rodriguez LA et al. Variability in risk of gastrointestinal complications with individual non-steroidal anti-inflammatory drugs: results of a collaborative meta-analysis. BMJ 1996;312:1563-6.

7. Garcia Rodriguez L, Cattaruzzi C, Troncon MG, Agostinis L. Risk of hospitalization for upper gastrointestinal drugs, calcium antagonists, and other antihypertensive drugs. Arch Intern Med 1998;158:33-9.

8. Chiroli S, Roggeri D. Consumo di risorse e costi per la diagnosi e la cura degli eventi avversi gastrointestinali dovuti all'uso dei FANS. Pharmacoeconomics - Italian Research Articles 2001; 3(2):61-70

9. Hogan DB, Campbell NRC, Crutcher R, Jennet P, MacLeod N. Prescription of nonsteroidal anti-inflammatory drugs for elderly people in Alberta. Can mad Assoc J 1994;151:315-322.

10. Blower AL, Brooks A, Fenn GC et al. Emergency admissions for upper gastrointestinal disease and their relaton to NSAID use. Aliment Pharmacol Ther 1997;12:283-291. 
11. Moore N, Montout C, Verschuren X, Callens J, Kong SX, Bégaud B. Use of gastrointestinal (GI) protective agents among patients on non-steroidal anti-inflammatory drugs in France and prevention or treatment of adverse GI effects. 12th EULAR Congress. Glasgow, Scotland, June 6-11, 1999. Rheumatol Europe 1999.

12. Lanas A. Impacto economico de los efectos secundarios gastrointestinales asociados a antiinflamatorios no esteroideos en el Servicio Nacional de Salud. Medicina Clinica 2000; vol. 114 (Sup. 3): 46 -53.

13. Langman MJ, Jensen DM, Watson DJ et al. Adverse upper gastrointestinal effects of rofecoxib compared with NSAIDs. JAMA 1999;282:1929-1933.

14. Laine L, Harper S, Simon T et al. A randomized trial comparing the effect of rofecoxib, a cyclooxygenase 2-specific inhibitor, with that of ibuprofen on the gastroduodenal mucosa of patients with osteoarthritis. Gastroenterology 1999 117:776-783.

15. Simon LS, Weaver AL, Graham DY et al. Anti-inflammatory and upper gastrointestinal effects of celecoxib in rheumatoid arthritis. JAMA 1999;282:1921-1928.

16. Silverstein FE, Faich G, Goldstein JL et al. Gastrointestinal Toxicity With Celecoxib vs Nonsteroidal Anti-inflammatory Drugs for Osteoarthritis and Rheumatoid Arthritis. The CLASS Study: A Randomized Controlled Trial. JAMA. 2000;284:1247-1255

17. Bombardier C, Laine L, Reicin A et al. Comparison of upper gastrointestinal toxicity of rofecoxib and naproxen in patients with rheumatoid arthritis. VIGOR Study Group. N Engl J Med. 2000 Nov 23; 343 (21): 1520-1528.

18. Haglund U, Svarvar P. The Swedish ACCES model: Predicting the health economic impact of celecoxib in patients with osteoarthritis or rheumatoid arthritis. Rheumatology 2000; 39(SUPPL. 2): 51-56.

19. Svarvar P, Aly A. Use of the ACCES model to predict the health economic impact of celecoxib in patients with osteoarthritis or rheumatoid arthritis in Norway. Rheumatology 2000; 39(SUPPL. 2): 43-50.

20. Pellissier JM, Straus WL, Watson D.J et al. Economic evaluation of rofecoxib versus noselective nosteroidal antiinflammatory drugs for the treatment of osteoarthritis. Clinical Therapeutics 2001;23(7), 1061-1079.

21. Soto J. Economic evaluation of diclofenac/misoprostol versus rofecoxib in the treatment of arthrosis. Atencion Farmaceutica. 2001;3(1):30-37.

22. Moore RA, Phillips CJ, Pellissier JM, Kong SX. Health economic comparisons of rofecoxib versus conventional nonsteroidal antiinflammatory drugs for osteoarthritis in the United Kingdom. J Med Economics 2001; 4, 1-17.

23. Marshall JK, Pellissier JM, Attard CL et al. Incremental cost-effectiveness analysis comparing rofecoxib with nonselective NSAIDs in osteoarthritis: Ontario Ministry of Health perspective. Pharmacoeconomics 2001; 19(10), 1039-1049.

24. Zabinski RA, Burke TA, Johnson J et al. An economic model for determining the costs and consequences of using various treatment alternatives for the management of arthritis in Canada. Pharmacoeconomics. 2001; 19 (Suppl 1): 49-58

25. Chancellor JVM, Hunsche E, de Cruz E, Sarasin FP. Economic evaluation of celecoxib, a new cyclo-oxygenase 2 specific inhibitor, in Switzerland. Pharmacoeconomics 2001; 19 (Suppl 1), 59-75.

26. Russo P, Attanasio E, Baio G et al. Una prima valutazione economica dell'impiego di rofecoxib vs FANS convenzionali nell'artrosi. PharmacoEconomics Italian Research Articles 2001;3:81-9.

27. You JH, Lee KK, Chan TY et al. Arthritis treatment in Hong Kong-cost analysis of celecoxib versus conventional NSAIDS, with or without gastroprotective agents. Aliment Pharmacol Ther 2002; 16(12): 2089-96.

28. Vargas E, Laredo L, Soto J, Rejas J. Economic evaluation of celecoxib in the treatment of arthrosis. AtencionFarmaceutica. 2002; 4(2): 72-82.

29. Weideman RA, Kelly KC, Kelley CL, Cryer B. COX-2-specific inhibitors: prescribing patterns in a large managed care health system and strategies to minimize costs. Am J Manag Care 2002; 8(10): 869-77.

30. Russo P, Capone A, Attanasio E et al. Pharmacoutilization and costs of osteoarthritis: changes induced by the introduction of a cyclooxygenase-2 inhibitor into clinical practice. Rheumatology 2003; 42: 258-266.

31. Consensus conference. Ruolo dei Coxib nel trattamento dell'artrosi. Reumatismo 2003; 55(Suppl. 1):6-48.

32. Lipsky PE. Reccomendations for the clinical use of cyclooxygenase-2-specific inhibitors. Am J Med 2001; 110(3A):3S$5 \mathrm{~S}$. 